\title{
Plasma Formation in the Near Anode Region in Hall Thrusters
}

\author{
Doug Browning, Michael Keidar and Iain D. Boyd \\ Department of Aerospace Engineering, University of Michigan, Ann Arbor MI 48109
}

\begin{abstract}
The plasma dynamics and ionization of propellant gas is modeled within the anode holes used for gas injection of a Hall thruster. Under conditions of anode coating with dielectric material, the discharge current must close within these holes which results in ionization and the formation of plasma jets emanating from the openings. The model shows an increase in electron current and a decrease of plasma potential as we look deeper into the holes. Therefore it is predicted that a "double layer" structure can exist in the near and interior anode regions with the total voltage drop as small as a few volts. This allows significant gain of electron energy and consequently strong ionization in the region of gas injection while reducing the detrimental effects on efficiency of the positive anode fall.
\end{abstract}

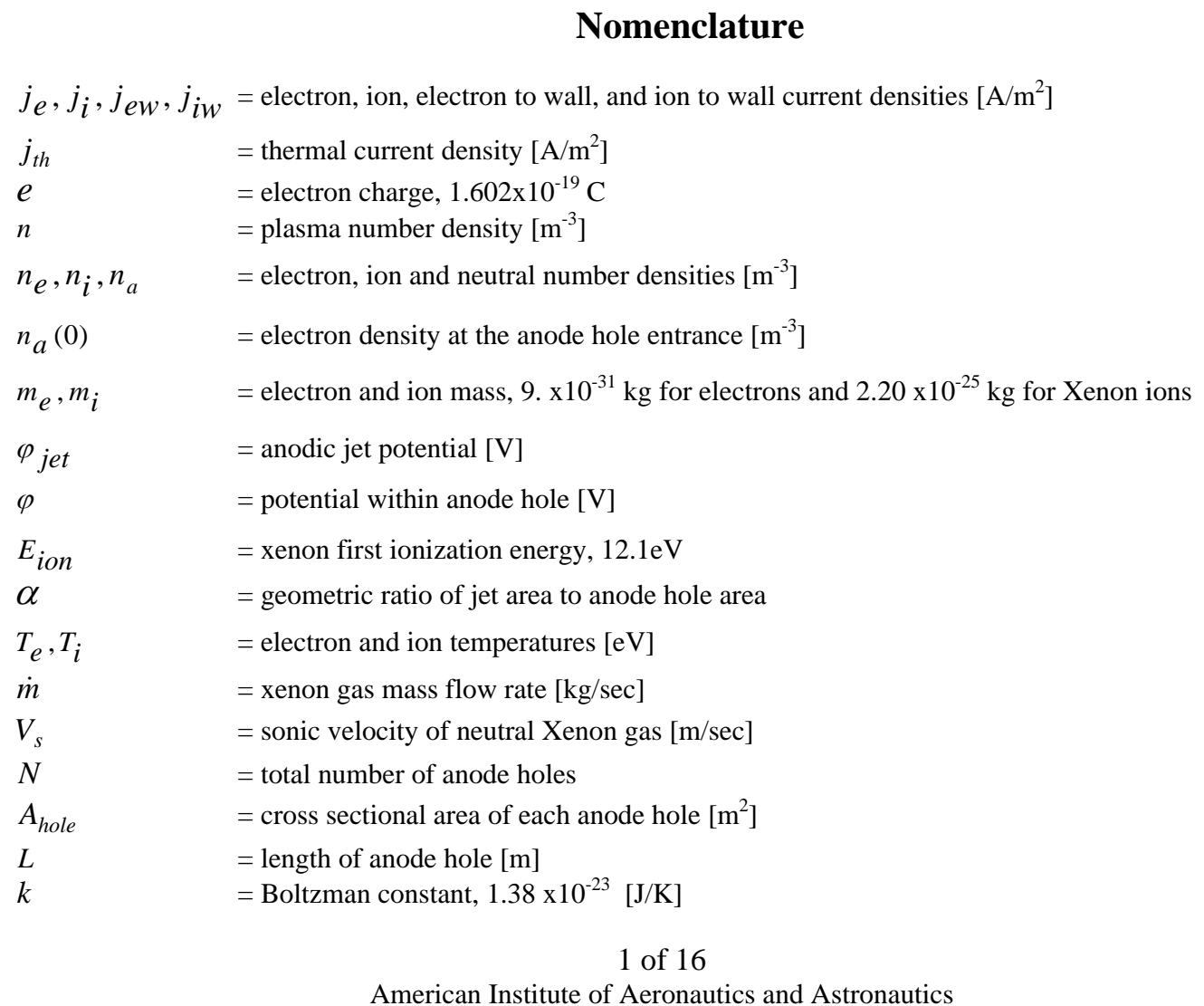


$n_{S} \quad=$ plasma density at internal anode sheath edge

\section{Introduction}

The Hall thruster is currently one of the most advanced and efficient types of electrostatic propulsion devices. The Hall thruster produces thrust density which is much higher than other types of stationary ion thrusters. This advantage comes from the fact that acceleration takes place in a quasi-neutral plasma and thus is not limited by space charge effects. State of the art Hall thrusters have an efficiency of about 50\% with specific impulses in the range of $1000 \mathrm{~s}-3000$ $\mathrm{s} .{ }^{1,2,3}$ The electrical discharge in the Hall thruster has a cross-field $(\mathrm{E} \times \mathrm{B})$ configuration where the external magnetic field is radial and perpendicular to the axial electric field, which accelerates the ions. The electron current passing across the magnetic field leads to an electron closed drift or Hall drift.

The transition plasma-wall region determines the particle and energy fluxes from the plasma to the wall ${ }^{4}$. One of the least studied boundary regions in the Hall thruster channel is the anode. In spite of considerable experimental and theoretical study of the internal structure of the Hall thruster, general understanding of the near anode phenomena is very limited. This is due in part to the fact that most of the existing models deal with plasma flow in the channel, plasma-wall interaction etc. assuming some boundary conditions at the anode. Additionally, it is exceedingly difficult to take measurements very close to or within the anode itself. However, the anode effects potentially play an important role in Hall thruster operation. Previously the near anode region was studied by Melikov. ${ }^{5,6} \mathrm{He}$ concluded that high-energy electrons penetrate from the channel into the anode through the holes and that electron-impact ionization begins in the anode cavity and anode holes. It was recently found that, dependent on the conditions at the anode, the anode voltage drop can be either positive or negative. ${ }^{7}$ While the case with the negative anode fall has been intensively studied, ${ }^{8,9}$ the positive anode fall is not generally understood. The negative anode fall is associated with the sonic transition for the ion flow and due to the fact that the electron thermal current in the near anode sheath is larger than the discharge current. As a result, there is a requirement for ion flow to the anode. In the case of the 
positive anode fall, electrons gain energy while moving towards the anode which results in higher power deposition at the anode and therefore possible drop in thruster efficiency.

In this paper a study is presented of the near anode region of the Hall thruster in the case of positive anode fall. It was found experimentally ${ }^{7}$ that this case is associated with anode coating and current closing through holes in the anode. These holes are used for gas injection into the thruster channel. It was shown that more stable operation of the Hall thruster can be achieved when the anode is in fact coated by a di-electric material. ${ }^{7}$ In this case, plasma is formed in the anode holes and creates a jet structure expanding downstream into the channel. In this work, we develop a model of the plasma formation within the anode holes as well as plasma expansion in the near anode region.

\section{Model Description}

In this section, a model is presented of electron behavior within the anode holes used for gas injection. Under conditions of anode di-electric coating, these holes behave similar to hollow anode ${ }^{10}$ and hollow cathode ${ }^{11}$ devices. Additional ionization can occur within this region. A description of the resulting effects on the current density and internal potential distribution will be attempted. Figure 1 shows the various plasma structures near to and within an individual anode hole. 


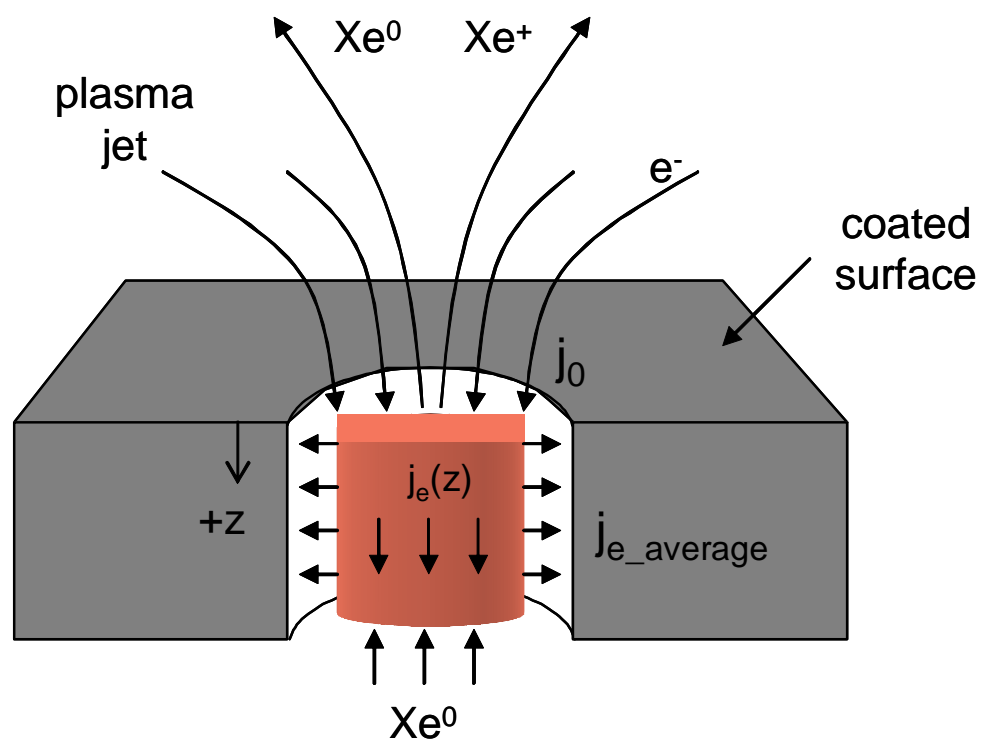

Figure 1: Anode hole plasma structure

As the electrons stream into the anode hole from the jet, they encounter neutral gas within the hole. Using xenon as the working gas, the ionization rate and subsequent impact on the electron current density is described by:

$\frac{d j_{e}}{d x}=e \beta n_{e} n_{a}$

Where: $\beta=10^{-20}\left(\frac{e \cdot \varphi_{\text {jet }}}{2 \pi \cdot m_{e}}\right)\left(3.97+0.64 \cdot \varphi_{\text {jet }}\right) \exp \left(\frac{-E_{\text {ion }}}{\varphi_{\text {jet }}}\right)$ (Ref. 11).

$n_{e}(0)=n_{e, 0} \frac{\sqrt{\alpha}}{\sqrt{\varphi_{j e t} / T_{e, 0}}}$

Electrons gain energy, $\varphi_{\text {jet }}$, in the plasma jet emanating from the gas injection holes (see next section). This energy will be used as the characteristic electron energy for ionization calculation. This potential drop also concentrates the electron density entering the hole. The total flux of electrons (and resulting electron current density) will increase deeper into the hole. Currently, a single electron temperature population is assumed for these calculations. 
The neutral gas density within the holes is described by the following:

$$
n_{a}=\frac{\dot{m} / N}{m_{i} \cdot V_{s} \cdot A_{\text {hole }}}
$$

Here a mass flow rate of $5 \mathrm{mg} / \mathrm{sec}$ and a neutral xenon sonic velocity at a gas temperature of $700 \mathrm{~K}^{12}$ is considered. These neutral densities turn out to be very high within the gas injection holes and strongly influence the degree of ionization.

A constant average current density closing within the anode hole is assumed that is dictated by the total discharge current, the number of holes and hole geometry. Because the plasma density changes within the hole due to ionization, the plasma sheath potential must change as well to maintain current continuity. This potential difference is described by the following:

$$
\begin{aligned}
& \Delta \varphi=-T_{e} \cdot \ln \left(\frac{j_{e}}{j_{a v}}\right) \\
& j_{a v}=\frac{I_{d}}{(2 \pi R L) \cdot N}
\end{aligned}
$$

This internal sheath structure, in conjunction with the jet potential, yields the total potential between the channel plasma and the anode surface.

Electron heating near the hole is calculated from the 2D model developed by Keidar et al. ${ }^{13}$ Briefly, the model is based on two-dimensional, axi-symmetric, two-fluid hydrodynamics that include the mass and momentum conservation for ions, neutrals and electrons. The following system of equations completely describes the plasma flow and current distribution:

$$
\begin{aligned}
& m_{i}\left(\overrightarrow{V_{i}} \cdot \vec{\nabla}\right) \vec{V}_{i}=-k\left(Z_{i} T_{e}+T_{i}\right) \cdot \vec{\nabla} \ln (n)+\frac{\vec{j} \times \vec{B}}{n}-v_{i a} m\left(\vec{V}_{i}-\vec{V}_{a}\right) \\
& \vec{j}=\sigma\left\{\vec{E}+\left(k T_{e} / e\right) \vec{\nabla} \ln (n)-\frac{\vec{j} \times \vec{B}}{e \cdot n}\right\}
\end{aligned}
$$




$$
\begin{aligned}
& \vec{\nabla} \cdot\left(\vec{V}_{\alpha} n_{\alpha}\right)=0 \\
& \vec{\nabla} \cdot \vec{j}=0
\end{aligned}
$$

Where $\vec{V}_{i}$ is the ion velocity, $\vec{E}$ is the electric field, $\vec{j}$ is the current density and $\vec{B}$ is the magnetic field (self or external). In this particular study a constant neutral velocity along the jet is assumed and the magnetic field effects neglected.

The formulation of the boundary conditions was described elsewhere ${ }^{13}$. The free plasma boundary is defined as the surface where the plasma jet velocity has only a tangential component, i.e., the normal component $V_{n}$ is zero. The plasma density at the free boundary of the plasma jet is $n=0$, and normal current density is $j_{n}=0$.

\section{Results and Discussion}

In this section, results are presented from the plasma model as it currently stands. These results represent a first attempt at describing the processes within the anode holes. Further refinement is needed to integrate the calculations into a single self-consistent model.

\subsection{Plasma Jet}

Some preliminary results of the anodic jet density structure are shown in Figure 2. Plasma is generated inside the anode hole and expands. The fast plasma jet expansion leads to plasma density decrease by a few orders of magnitude at a distance of a few mm from the hole. Figure 3 shows the jet voltage as a function of electron density to neutral density ratio at the exit plane. It can be seen that a potential drop in the range of 10 to 30 volts is possible under these conditions. 


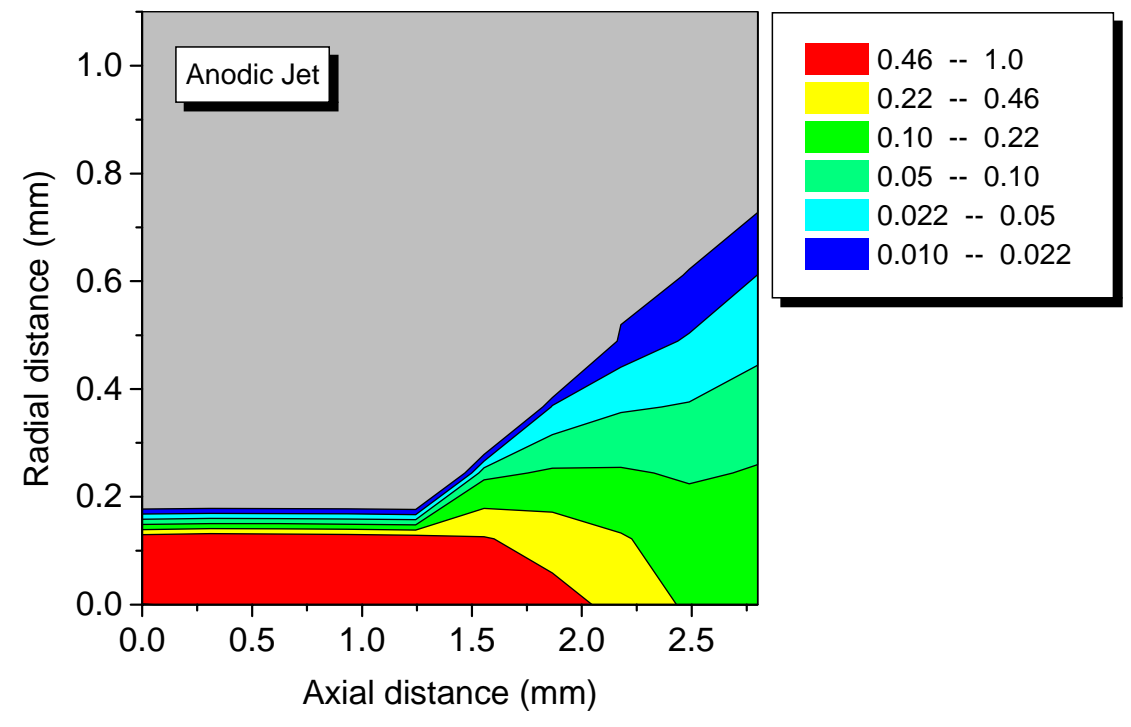

Figure 2: Plasma density distribution near a single anode hole.

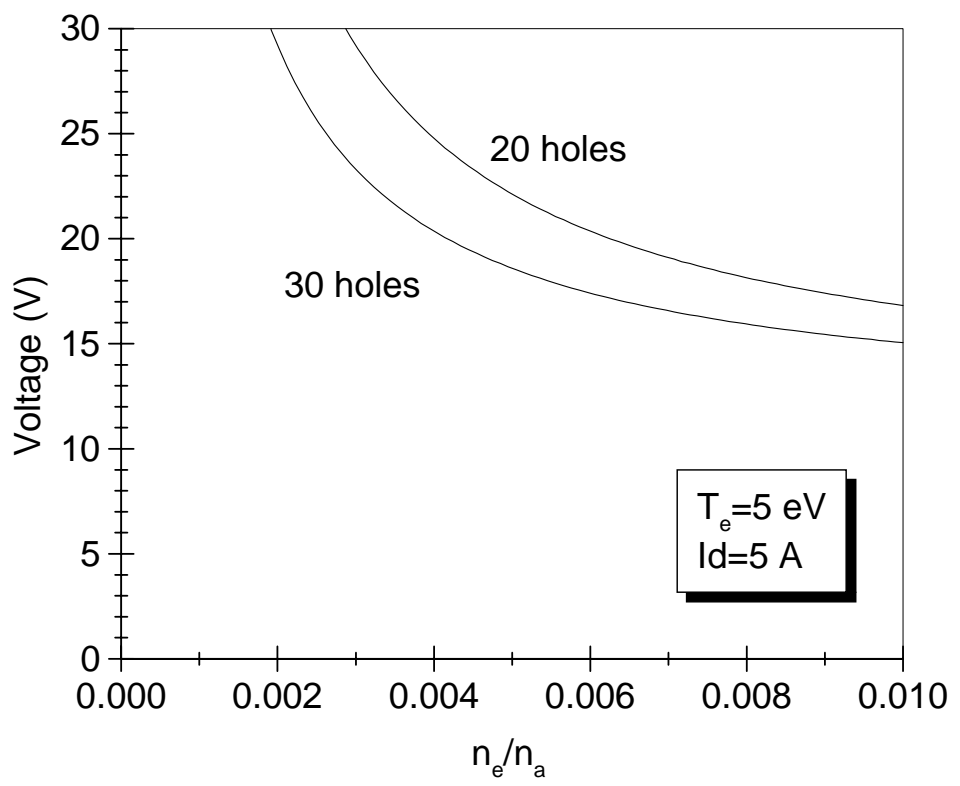

Figure 3: Anode jet voltage as a function of electron density to neutral density ratio. The anode hole radius is $0.1 \mathrm{~mm}$. 


\subsection{Ionization and Current Density}

Electrons entering the anode hole produce significant ionization of the gas. As a result, the electron current increases along the hole as shown in Figure 4. Here we assumed a discharge current of 5 Amps with anode hole radius of $0.1 \mathrm{~mm}$. The current is shared equally among 20 gas injection holes. The initial electron density is $2 \times 10^{16} \mathrm{~m}^{-3}$ with an electron temperature of $5 \mathrm{eV}$. The energy necessary for ionization is gained in the anodic jet region (shown in Figure 2) and depends on the voltage drop along the anodic plasma jet as shown in Figure 3.

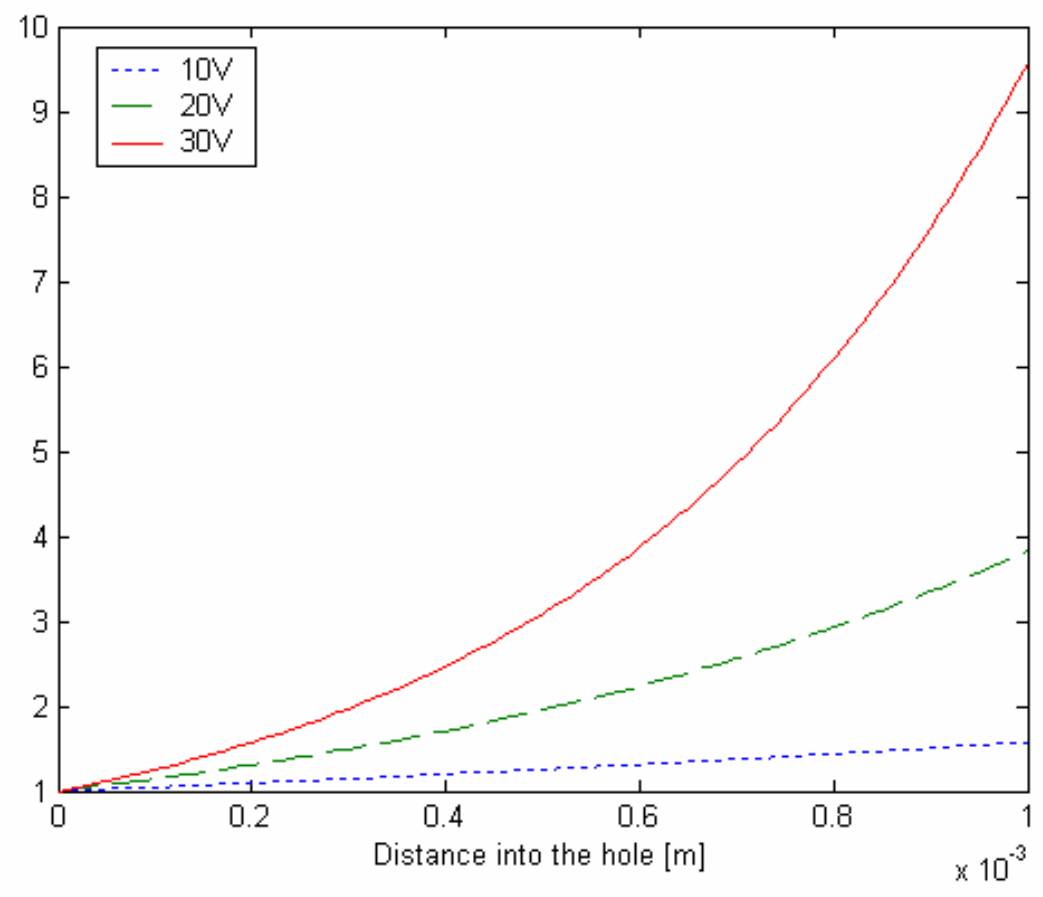

Figure 4: Electron current (normalized to initial current density entering the hole) increase along the anode hole with potential drop in the anodic jet as a parameter

The potential drop in the hole is negative due to excess of electron current. The potential distribution within the hole is shown in Figure 5. The decrease in potential allows the average current density going to the anode wall to remain constant as the electron density 
is increasing due to ionization. This is an important condition for maintaining current continuity. The total potential drop between the channel plasma and the anode hole wall is shown in Figure 6. It can be seen that this total potential drop is lower than the jet potential voltage. Therefore, it is predicted that a double layer structure can exist in the near and interior anode region with the total voltage drop as small as a few volts. This allows significant gain of electron energy and consequently strong ionization in the region of gas injection while reducing the detrimental efficiency effects of the positive anode fall.

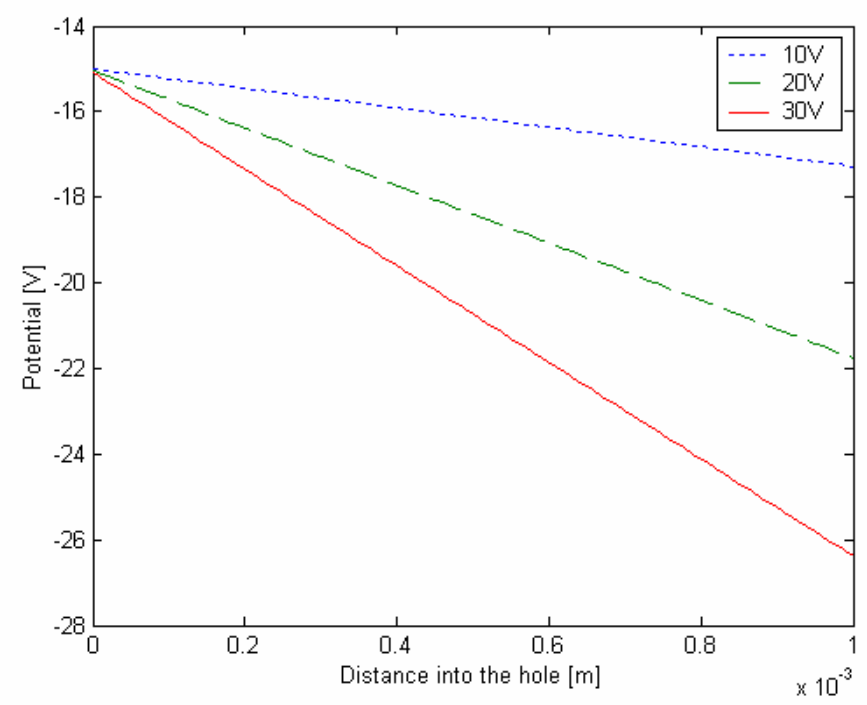

Figure 5: Potential drop (in V) between the plasma and the wall inside the anode hole with potential drop in the anodic jet as a parameter. 


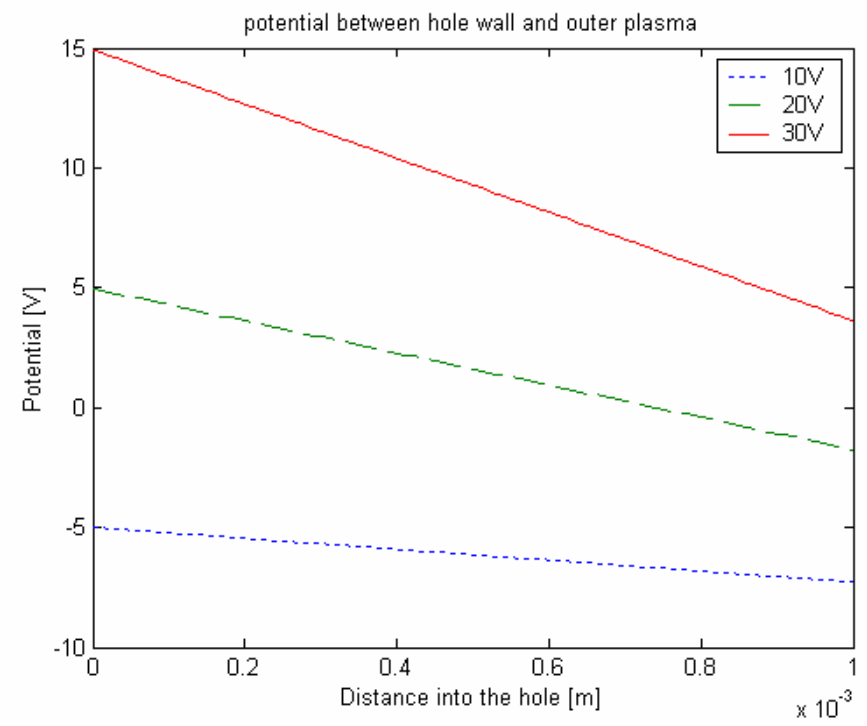

Figure 6: Potential drop (in V) between the channel plasma and the wall inside the anode hole with potential drop in the anodic jet as a parameter.

In addition to this baseline case, several variations in input parameters are investigated using the model. The primary variations are geometric parameters of the anode holes and a range of initial electron densities. The results for these cases are tabulated in Table 1 below.

Table 1: Results for several input variations

\begin{tabular}{|c|c|c|c|c|c|c|c|c|}
\hline \multirow{2}{*}{$\begin{array}{c}\text { Initial Electron } \\
\text { Density } \\
{\left[\mathrm{m}^{\wedge}-3\right]} \\
\end{array}$} & \multirow{2}{*}{$\begin{array}{c}\text { Anode Hole } \\
\text { Radius } \\
{[\mathrm{m}]}\end{array}$} & \multirow{2}{*}{$\begin{array}{c}\text { Number of } \\
\text { Holes } \\
\mathrm{N}\end{array}$} & \multicolumn{3}{|c|}{$\begin{array}{l}\text { Maximum Current Density Increase } \\
\text { Factor for Each Jet Voltage }\end{array}$} & \multicolumn{3}{|c|}{$\begin{array}{l}\text { Potential Between Outer Plasma and } \\
\text { Bottom of Anode Hole Wall [V] }\end{array}$} \\
\hline & & & $10 \mathrm{~V}$ & $20 \mathrm{~V}$ & $30 \mathrm{~V}$ & $10 \mathrm{~V}$ & $20 \mathrm{~V}$ & $30 \mathrm{~V}$ \\
\hline \multirow[t]{4}{*}{$2.0 \mathrm{E}+16$} & 1.0E-04 & 20 & 1.2 & 1.6 & 2.15 & -6 & 2.5 & 11 \\
\hline & $2.0 \mathrm{E}-04$ & 20 & 1.2 & 1.6 & 2.15 & -2.5 & 6.5 & 14.5 \\
\hline & 1.0E-04 & 30 & 1.2 & 1.6 & 2.15 & -6 & 2.5 & 11.5 \\
\hline & 2.0E-04 & 30 & 1.2 & 1.6 & 2.15 & -2.5 & 6.5 & 15 \\
\hline \multirow[t]{4}{*}{$6.0 \mathrm{E}+16$} & 1.0E-04 & 20 & 1.6 & 3.8 & 9.5 & -7.5 & -2 & 4 \\
\hline & 2.0E-04 & 20 & 1.6 & 3.8 & 9.5 & -3.5 & 2 & 7.5 \\
\hline & 1.0E-04 & 30 & 1.6 & 3.8 & 9.5 & -7.5 & -2 & 3.5 \\
\hline & 2.0E-04 & 30 & 1.6 & 3.8 & 9.5 & -3.5 & 2 & 7.5 \\
\hline \multirow[t]{4}{*}{$1.0 \mathrm{E}+17$} & 1.0E-04 & 20 & 2.5 & 9 & 42 & -8.5 & -6.5 & -3.5 \\
\hline & 2.0E-04 & 20 & 2.5 & 9 & 42 & -5 & -2.5 & 0 \\
\hline & 1.0E-04 & 30 & 2.5 & 9 & 42 & -8.5 & -6.5 & -3.5 \\
\hline & 2.0E-04 & 30 & 2.5 & 9 & 42 & -5 & -2.5 & 0 \\
\hline
\end{tabular}


It is interesting to note that the normalized current density increase within each hole is not sensitive to the geometric variations. The potential structure does change for geometric variations but is much more sensitive to the total number of anode holes than the hole size. It is possible that this trend is merely a function of the calculation method. Currently the anode jet structure calculation is not sensitive to the hole size. Therefore, we may not be adequately taking into account other effects that precipitate from varying the hole geometry.

Furthermore, there are cases where the calculated potential starts positive at the beginning of the hole and decreases until it becomes negative. This phenomenon, although possibly real, is most likely due to the calculation method as well. These cases seem to reveal situations where the simplifying assumptions (such as assumption about average current density in the hole) used for the potential calculation are no longer valid. During these conditions, the discharge current is likely collected either in just the front part of the hole or continues through the hole and closes to the back side of the anode within the gas distribution area as shown schematically in Figure 7. Either case would result in a change in the effective current collecting surface area which is different from the hole geometry. In order to address these issues, a model is developed for the current distribution along the anode hole, which is described in the next section.

\subsection{Current distribution in the anode hole}

Up to this point most of the focus has been on the electron influence of the internal potential structure. However, an understanding of the contributions from each plasma species is important to describing the internal plasma structure. A current conservation equation is employed which reads:

$j_{d}=j_{i}+j_{e}-j_{e w}+j_{i w}$ 
Where $\mathrm{j}_{\mathrm{d}}$ is the discharge current density, $\mathrm{j}_{\mathrm{e}}$ is the electron current density, $\mathrm{j}_{\mathrm{i}}$ is the ion current density, $\mathrm{j}_{\mathrm{ew}}$ is the electron current density to the wall and $\mathrm{j}_{\mathrm{iw}}$ is the ion current density to the wall. Schematically, this balance is shown in Figure 7.

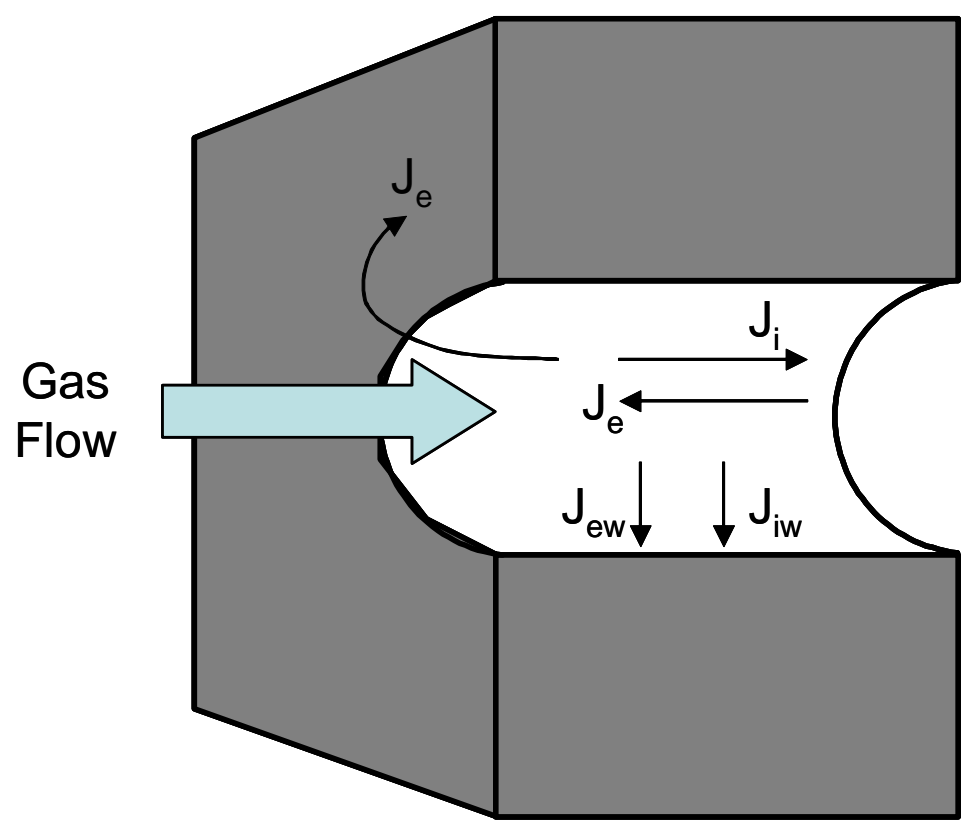

Figure 7: Current density schematic

The electron current density to the wall can be calculated using:

$$
j_{e w}=j_{t h} \exp \left(-\frac{\Delta \varphi}{T_{e}}\right)
$$

Ion current density at the wall can be calculated using the Bohm condition:

$$
j_{i w}=e n_{s} \sqrt{\frac{T_{e}}{m_{i}}}
$$

In the diffusion approximation, ${ }^{11}$ the axial component of the electron and ion velocities can be calculated using corresponding expressions for mobilities. 
The current densities distribution is shown in Figure 8. As is clearly shown, the electron current density dominates which is expected given their higher mobility. The ion current density starts strong at the beginning of the hole but drops off rather quickly. In addition it should be pointed out that the resulting potential distribution within the hole may lead to ion backflux. The current collected by the anode hole wall depends on the initial electron energy which is a function of the potential drop along the anodic jet (as discussed in Sec. 3.1). The dependence of the wall current on the total current ratio used for the total potential drop within the hole is shown in Figure 9. One can see that in the case of the small potential drop, only a fraction of the current is closed within the hole. The current can be closed to the back side of the anode as shown schematically in Figure 7. According to this model, the predicted current collected by the wall will approach the total current when the anode voltage is about $15 \mathrm{~V}$.

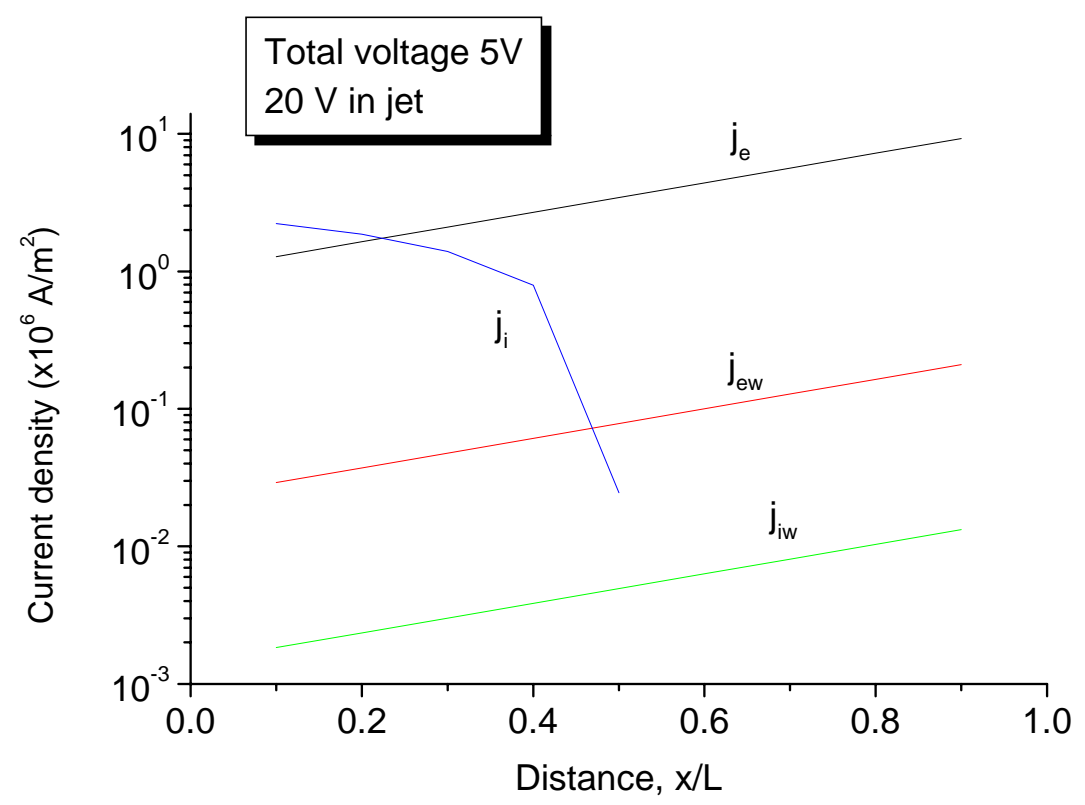

Figure 8: Current density distribution within the anode holes 


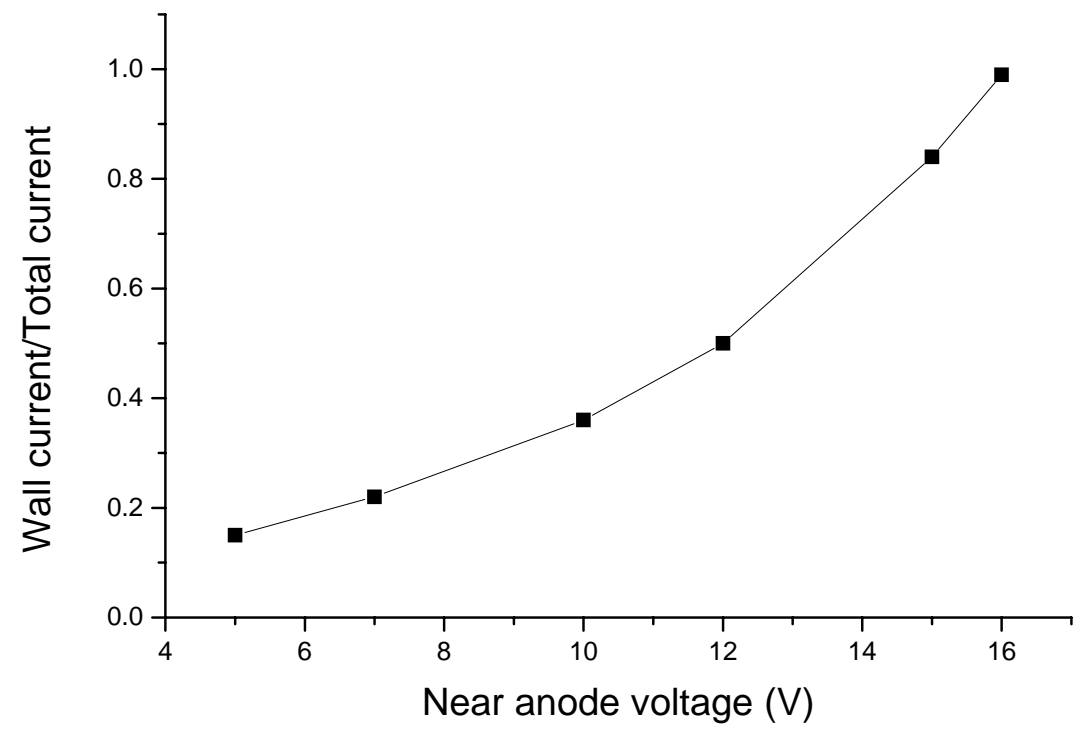

Figure 9: Fraction of wall current within the anode hole as a function of the total anode voltage

\section{Concluding Remarks}

This preliminary investigation has yielded useful insight into the internal ionization and potential structure of the anode region of a di-electric coated Hall thruster. Anode coating during operation is a common occurrence for Hall thrusters. The fact that this phenomenon is also linked to a "quieting" of the discharge oscillation provides additional incentive for further study. Eventually, understanding of these processes may yield improved thruster designs with less noise in the discharge. To date we are unaware of any measurements having been taken inside the anode holes. Experimental data within these holes is necessary to verify the assumptions made in this work. Needless to say, such a task would be very difficult given the tiny scale.

The baseline case shows that for nominal operation during conditions of anode coating there is as much as a factor of 10 increase in electron current density within the anode holes due to ionization. The resulting potential structure within the holes for this case yields a positive anode fall of roughly $4 \mathrm{~V}$ between the bottom of the anode hole wall and 
the near anode plasma ( $\sim 3 \mathrm{~mm}$ from the anode). This potential is much less than the $30 \mathrm{~V}$ potential through the anodic jets emanating from the holes. This “double layer” structure allows significant gain of electron energy and consequently strong ionization within the holes while yielding a much lower total anode fall.

Variations of anode hole geometry and the number of holes had little impact on the relative increase of current density but did affect the potential distribution within the holes. Of the input parameter variations that were studied, the largest influence came from the initial electron density. This makes sense given the close coupling of electron density to the degree of ionization within the holes. Furthermore, several flaws in the simplifying assumptions were visible in many of the cases. The potential structure calculations within the holes were based on the physical parameters of the anode holes. Under certain conditions, the current closure likely deviates from this physical limitation. Future refinement of the assumptions and level of integration within the code are necessary to solve these issues. The next step is to create a self-consistent analysis of the current distribution within the hole and gas distributor as well as coupling of the anode hole ionization model with the 2D model of the anodic jet.

\section{Acknowledgements}

The authors wish to thank Y. Raitses, L. Dorf, I. Katz, and D. Jacobson for very useful discussions on this subject. The MK and IB gratefully acknowledge the financial support by the Air Force Office of Scientific Research, Grant No FA9550-05-1-0042.

\section{REFERENCES}

${ }^{1}$ V. Kim, J. Prop. Power, 14, 736 (1998)

${ }^{2}$ V.V. Zhurin, H.R. Kaufman and R.S. Robinson, Plasma Sources Sci. Technol., 8, 1 (1999)

${ }^{3}$ A.I. Morozov and V.V. Savelyev, in Review of Plasma Physics, Ed. By B.B. Kadomtsev and V.D. Shafranov (Consultant Bureau, New York, 2000), Vol. 21, p. 203. 
${ }^{4}$ M. Keidar, I.D. Boyd and I.I. Beilis, "Plasma flow and plasma-wall transition in Hall thruster channel”, Phys. Plasmas, vol. 8, No. 12, pp. 5315-5322, (2001).

${ }^{5}$ Melikov, I., "Point and Jet Ionization in a Hall Plasma Accelerator," Soviet Phys. Tech. Phys., Vol. 22, No 4, 1977, pp. 452-453

${ }^{6}$ Melikov I.V. "Experimental investigation of anode process in a closed electron drift accelerator,” Soviet Phys. Tech. Phys., Vol. 19, No 1, 1974, pp. 35-37.

${ }^{7}$ Dorf, L., Y. Raitses and N.J. Fisch., "Effect of Anode Dielectric Coating on Hall Thruster Operation”, Applied Physics Letters, Vol. 84 No. 7, 2004, 1070

${ }^{8}$ M. Keidar, I.D. Boyd and I.I. Beilis, “Analysis of the Anode Region of a Hall Thruster Channel,” AIAA-2002-4107, July 2002

${ }^{9}$ J. Koo, M. Keidar and I.D. Boyd, "Boundary Conditions for a 2-D Hybrid Stationary Plasma Thruster Model,” AIAA-2004-3781, July 2004

${ }^{10}$ Anders, A., Anders, S., “The Working Principal of the Hollow Anode Plasma Source," Plasma Sources Science Technology, 4(1995), pp. 571-575

${ }^{11}$ I. Katz, J.R. Anderson, J.E. Polk, and J. R. Brophy, One-dimensional hollow cathode model, J. Prop. Power, 19, No. 4, 2003, pp. 595-600.

${ }^{12}$ Dorf, L., Y. Raitses and N.J. Fisch, "Experimental Studies of Anode Sheath Phenomena in a Hall Thruster Discharge,” Journal of Applied Physics, 97, 103309, 2005

${ }^{13}$ M. Keidar, I. Beilis, R. L. Boxman, and S. Goldsmith., "2-D Expansion of the lowdensity interelectrode vacuum arc plasma jet in an axial magnetic field,”. J. Phys. D: Appl. Phys., vol. 29, 1996, pp. 1973-1983 